\title{
Partizipative Forschung im Übergang von der Pflichtschule in weitere schulische Bildung, Ausbildung oder Beruf
}

\author{
Helga Fasching \\ Universität Wien
}

\begin{abstract}
Im Fokus des Themenstrangs ,Inklusion im Übergang von der Pflichtschule in weitere schulische Bildung, Ausbildung oder Beschäftigung“" stehen verschiedene Zugänge zur Erforschung von inklusiven Übergangsprozessen. Die bereits veröffentlichten Beiträge des Themenstranges konnten unter anderem die Bedeutung von intersektionalen, längsschnittlichen und biografiebezogenen Betrachtungsweisen aufzeigen (Demmer 2017; Fasching 2016). Der vorliegende Beitrag widmet sich der partizipativen Forschung, einem Forschungszugang, der im deutschsprachigen Raum noch wenig verbreitet ist, von dem jedoch zu hoffen ist, dass er in Zukunft weiter an Bedeutung gewinnt.
\end{abstract}

\section{Einleitung}

In den letzten Jahrzehnten waren Übergänge innerhalb der Bildungswissenschaft Gegenstand zahlreicher theoretischer, aber auch empirischer Diskussionen. Durch die intensive und systematische Beschäftigung konnte sich eine umfassende Übergangsforschung etablieren, deren Erkenntnisinteresse darin besteht, Phänomene zu untersuchen, die mit einschneidenden bzw. nachhaltigen Veränderungen, Umstrukturierungen oder einem Wechsel verbunden sind (Thielen 2011, 9f.). Obwohl eine Vielzahl von Ereignissen im menschlichen Lebenszusammenhang - etwa eine Erkrankung, ein Umzug oder eine Familiengründung - solche Phänomene darstellen können, liegt der Fokus in der bildungswissenschaftlichen Übergangsforschung seit jeher stark auf spezifischen Übergängen innerhalb des institutionalisierten Bildungssystems (Tillmann 2013, 17). $\mathrm{Da}$ (Aus-)Bildung und Erwerbsarbeit als wichtige Voraussetzungen für gesellschaftliche Teilhabe gelten, stellt der Übergang von der Schule in weitere Bildung, Ausbildung und Beruf ein Thema dar, dem - sowohl in der Bildungswissenschaft als auch in den Subdisziplinen der Sonder- und Heilpädagogik bzw. der Inklusiven Pädagogik - traditionell vergleichsweise viel Aufmerksamkeit zuteil wurde.

Wie in der theoretischen und empirischen Literatur vielfach bemerkt, zeichnen sich Übergänge generell durch ihre Funktion als „sozial strukturierte Verteilerpunkte" (Wolter 2013, 45) aus. So wirken in ihnen nicht nur (verstärkt) bestehende soziale Ungleichheiten, sondern es finden auch weitere Inklusions- und Exklusionsprozesse statt, die die soziale Selektion vorantreiben können (Bellenberg/Forell 2013, 9; Solga 2009, 6). Für benachteiligte und gefährdete Personengruppen besitzt der Übergang in das Ausbildungs- und Erwerbssystem somit einen besonders risikoreichen und unsicheren Charakter. Sehr eindrücklich zeigt sich dies an den Übergangsverläufen von Jugendlichen mit Behinderungen, die sich häufig besonders schwierig gestalten, mit überdurchschnittlich vielen 
Irr- und Umwegen sowie Rückschlägen verbunden sind und sich ohne umfassende Unterstützung in der Regel kaum meistern lassen. Hieraus ergeht schließlich ein klarer Auftrag an die inklusionspädagogische Übergangsforschung, sich näher mit dem spezifischen Übergang von der Schule in weitere Bildung, Ausbildung oder Beruf zu befassen. Dabei geht es nicht allein um eine tiefgehende Untersuchung der konkreten Übergangswege, Übergangserfahrungen und Bewältigungsstrategien von Menschen mit Behinderungen, sondern vor allem auch um die Identifikation von begünstigenden und benachteiligenden Kontextfaktoren. Um diesem Auftrag nachkommen zu können, bedarf es jedoch angemessener Forschungsansätze. Der vorliegende Beitrag beleuchtet den partizipativen Forschungsansatz und plädiert für dessen Fruchtbarmachung für die inklusionspädagogische Übergangsforschung.

\section{Partizipative Forschung}

Sonder- und heilpädagogische Forschung war traditionellerweise Forschung über statt Forschung mit Menschen mit Behinderungen. Die eng mit aktivistischen Diskursen um das soziale Modell von Behinderung verbundene Forderung nach einer stärkeren Beteiligung von Betroffenen im Forschungsprozess fand erstmals in den 1970er Jahren Zuspruch und erlebt seit den 1990er Jahren einen neuen Aufschwung (Buchner u. a. 2011, 5; Unger 2014, 3ff.). Der Begriff der „inklusiven“ bzw. „partizipativen Forschung" verweist dabei heute auf eine Reihe von Ansätzen, bei denen Menschen mit Behinderungen als Mit-Forschende an der akademischen Wissensproduktion beteiligt sind, wobei sich die Ansätze hinsichtlich des Ausmaßes der Einbeziehung und Mitbestimmung deutlich unterscheiden können (Bigby u. a. 2014). Das gemeinsame Grundanliegen dieser Ansätze ist es, Menschen mit Behinderungen Gehör zu verschaffen und ihnen die Möglichkeit zu geben, ihre Sichtweisen in die Theoriebildung einzubringen.
Interessanterweise blieben nicht nur die Sichtweisen von Menschen mit Behinderung, sondern auch jene von deren Familien lange Zeit weitestgehend auf der Strecke, obwohl Familienmitglieder mit ihrem Wissen ebenfalls eine wertvolle Ressource für die Theoriebildung darstellen können (Whitson/Keller 2004; Lindstrom u. a. 2007; Turnbull u. a. 2011). Insbesondere das Image von Eltern hat sich jedoch in den letzten vierzig Jahren stark gewandelt. Während Eltern in den 1970er Jahren noch als „Problem“ und als „Verursacher" von Störungen bei einem Familienmitglied gesehen wurden (Evans 1975; Todd 2007), lässt sich seit den 1990er Jahren eine Abkehr von diesem unangemessenen Ursache-Wirkungs-Denken und eine Schwerpunktsetzung auf den Gesichtspunkt der „Familie als Ressource“ beobachten (Cunningham/Davis 1985; BüschgesAbel 2000). Darüber hinaus kamen im angloamerikanischen Sprachraum seit Mitte der 1980er Jahre vermehrt qualitative Forschungsmethoden zum Einsatz, um die Perspektiven von Menschen mit Behinderung und ihren Familien einzubeziehen (Atkinson u. a. 2001; Knox u. a. 2000; Morningstar u. a. 1995; Turnbull/Turnbull 1997; Turnbull u. a. 2011; Wolfendale 1997; Walmsley/Johnson 2003). Trotz dieser Forschungsbemühungen wurde die Kooperation zwischen Eltern und Professionellen jedoch tendenziell als entmachtend und im Hinblick auf bereits exkludierte bzw. marginalisierte Familien als bestehende Ungleichheiten verstärkend kritisiert (Bacon/CaustonTheoharis 2013; Morningstar u. a. 1995; Trainor 2005; Todd 2007; Turney/Kao 2009).

\section{Fazit}

Aus diesen Schilderungen und Befunden lassen sich zwei wichtige Hinweise für die partizipative Erforschung des Übergangsprozesses an der Schnittstelle von der Schule in das Ausbildungs- oder Erwerbssystem ableiten: 


\section{Partizipative Forschung im Übergang von der Schule in weitere Bildung, Ausbildung und Beruf stellt die Jugendlichen mit Behinderung in den Mittelpunkt!}

Die inklusionspädagogische Übergangsforschung kann zwar durchaus davon profitieren, möglichst viele an der Gestaltung dieses Überganges beteiligte Akteure, wie beispielsweise Eltern und Erziehungsberechtigte, an der Wissensproduktion partizipieren zu lassen, doch sollte die Partizipation von Menschen mit Behinderung stets im Zentrum stehen und nicht $\mathrm{zu}$ einem reinen Lippenbekenntnis verkommen (vgl. Todd 2007).

\section{Partizipative Forschung im Übergang von der Schule in weitere Bildung, Ausbildung und Beruf ist selbstreflexiv und selbstkritisch!}

Die inklusionspädagogische Forschung sollte sich das Ziel setzen, Diskriminierungsverhältnisse aufzudecken und abzubauen statt sie $\mathrm{zu}$ reproduzieren. Insbesondere muss sie in diesem Zusammenhang dafür Sorge tragen, dass die Stimmen der jungen Frauen und Männer mit Behinderung nicht untergehen und dass ihre Partizipation an der Theoriebildung einen ermächtigenden statt entmachtenden Charakter annimmt.

\section{Literatur}

Atkinson, M.; Wilkin, A.; Stott, A.; Kinder, K. (2001): Multi-Agency Working: An Audit of Activity. LGA Research Report 17. Slough: NFER

Bacon, J. K.; Causton-Theoharis, J. (2013): 'It should be teamwork': a critical investigation of school practices and parent advocacy in special education. In: International Journal of Inclusive Education 17, 682-699. https://doi.org/10.108 o/13603116.2012.708060

Bellenberg, G.; Forell, M. (2013): Einleitung. In: Bellenberg, G.; Forell, M. (Hrsg.): Bildungsübergänge gestalten. Ein Dialog zwischen Wissenschaft und Praxis. Münster: Waxmann, 9-13
Bigby, C.; Frawley, P.; Ramcharan, P. (2014): Conceptualizing inclusive research with people with intellectual disability. In: Journal of Applied Research in Intellectual Disabilites 27, 3-12. https://doi.org/10.1111/jar.12083

Buchner, T.; Koenig, O.; Schuppener, S. (2011): Gemeinsames Forschen mit Menschen mit intellektueller Behinderung. Geschichte, Status quo und Möglichkeiten im Kontext der UN-Behindertenrechtskonvention. In: Teilhabe 50 (1), 4-10

Büschges-Abel, W. (2000): Systemische Beratung in Familien mit behinderten oder chronisch erkrankten Angehörigen. Ein lösungsorientierter Ansatz für Heilpädagogik und klinische Sozialpädagogik. Neuwied: Luchterhand

Cunningham, C.; Davis, H. (1985): Working with Parents. Frameworks for Collaboration. Buckingham: Open University Press

Demmer, C. (2017): Ein Schritt nach vorn - ein Blick zurück. Biografieanalytische und intersektionale Betrachtungen von institutionellen Übergängen nach der Schule. In: Vierteljahresschrift für Heilpädagogik und ihre Nachbargebiete 86, 13-25. https://doi.org/10.2378/vhn20 17.arto2d

Evans, R. (1975): Children at risk: identification before school. In: Aspects of Education 20, 10- 23 Fasching, H. (2016): Nachschulische Arbeits- und Lebenssituation von jungen Frauen und Männern mit intellektueller Beeinträchtigung in Österreich. Eine Verbleibs- und Verlaufsstudie fünf Jahre nach Beenden der Schule. In: Vierteljahresschrift für Heilpädagogik und ihre Nachbargebiete 85, 290-306. https://doi.org/ 10.2378/vhn2016.art38d

Knox, M.; Mok, M.; Parmenter, T. (2000): Working with the experts: Collaborative research with people with an intellectual disability. In: Disability \& Society 15, 49-61. https://doi.org/10. $1080 / 09687590025766$

Lindstrom, L.; Doren, B.; Metheny, J. (2007): Transition to employment: Role of the family in career development. In: Exceptional Children 73, 348-366. https://doi.org/10.1177/0014402 90707300305

Morningstar, M.E.; Turnbull, A.P.; Turnbull, H.R. (1995): What do students with disabilities tell us about the importance of family involvement in the transition from school to adult life? In: Exceptional Children 62, 249-260 
Solga, H. (2009): Biographische Sollbruchstellen. Übergänge im Lebensverlauf bergen Chancen und Risiken. In: WZB-Mitteilungen 123, 6-7. Online: https://www.wzb.eu/sites/default/files/ publikationen/wzb_mitteilungen/wm123einzel6-7.pdf, 3.4. 2017

Thielen, M. (2011): Pädagogik am Übergang. Einleitende Gedanken zu Übergängen, Übergangsgestaltung und Übergangsforschung. In: Thielen, M. (Hrsg.): Pädagogik am Übergang. Arbeitsweltvorbereitung in der allgemeinbildenden Schule. Bad Heilbrunn: Klinkhardt, 8-19

Tillmann, K.-J. (2013): Einführung. Die Bewältigung von Übergängen im Lebenslauf - eine biografische Perspektive. In: Bellenberg, G.; Forell, M. (Hrsg.): Bildungsübergänge gestalten. Ein Dialog zwischen Wissenschaft und Praxis. Münster: Waxmann, 15-32

Todd, L. (2007): Partnerships for Inclusive Education. A Critical Approach to Collaborate Working. London, New York: Routledge

Trainor, A. (2005): Self-determination perceptions and behaviors of diverse students with LD during transition planning process. In: Journal of Learning Disabilities 38, 233-249. https://doi. org/10.1177/00222194050380030501

Turnbull, A.P.; Turnbull, H.R. (1997): Families, Professionals, and Exceptionality: A Special Partnership. 3rd Edition. New Jersey: Pearson

Turnbull, A.P.; Turnbull, H.R.; Erwin, E.J. (2011): Families, Professionals, and Exceptionality: Positive Outcomes through Partnerships and trust. 6th Edition. New Jersey: Pearson

Turney, K.; Kao, G. (2009). Barriers to school involvement: Are immigrant parents disadvantaged? In: Journal of Educational Research 102,
257-270. https://doi.org/10.3200/joer.102.4.2 57-271

Unger, H.v. (2014): Partizipative Forschung. Einführung in die Forschungspraxis. Wiesbaden: Springer

Walmsley, J.; Johnson, K. (2003): Inclusive Research with People with Learning Disabilities, Past, Present and Future. London: Jessica Kingsley

Whitson, V.; Keller, B. (2004): The influence of family of origin on career development: a review and analysis. In: The Counselling Psychologist 32, 493-568. https://doi.org/10.1177/0011000 004265660

Wolfendale, S. (1997): Working with Parents or SEN Children After the Code of Practice. London: David Fulton

Wolter, A. (2013): Übergang aus dem Schulsystem heraus. Übergänge zwischen Schule, beruflicher Bildung und Hochschule - Entwicklungen und Herausforderungen aus der Sicht der empirischen Bildungsforschung. In: Bellenberg, G.; Forell, M. (Hrsg.): Bildungsübergänge gestalten. Ein Dialog zwischen Wissenschaft und Praxis. Münster: Waxmann, 45-61

\section{Anschrift der Autorin}

\section{Assoz. Prof. Mag. Dr. Helga Fasching}

Vize-Studienprogrammleitung SPL 19 Institut für Bildungswissenschaft

Universität Wien

Sensengasse 3 a

A-1090 Wien

Tel.: +431427746803

helga.fasching@univie.ac.at 